\title{
Effect of gastrectomy on biliary dynamics
}

\author{
A. P. WYATT
}

From the War Memorial Hospital, Woolwich, London

A recent study of the physiology of flow through the sphincter of Oddi (Wyatt, 1967) indicated that amongst other factors gastric peristalsis was related to changes of resistance at the sphincter. This was especially notable when food was present in the stomach. Electrical stimuli leading to contraction of the gastric antrum often resulted in relaxation of the sphincter with increased flow through it. This relationship did not depend on extrinsic nervous connexions and was therefore supposed to depend on the continuity of smooth muscle of the stomach, duodenum, and sphincter of Oddi, or on the intrinsic nerve plexus of the bowel in this region. This raises the question as to what effect, if any, there is on bile flow when the stomach is disconnected from the duodenum as in a Polya type of gastrectomy. Further experiments are now reported which show that there are changes in biliary flow and pressure patterns after this type of operation.

\section{EXPERIMENT 1}

An acute experiment was set up, precisely as described previously (Wyatt, 1967), except that the dog was purposely fed a meal of mincemeat just before being anaesthetized. Gastric and duodenal pressures were monitored by balloons placed in the gastric antrum and the second part of the duodenum. Flow through the sphincter of Oddi was measured by a drop counter placed in the inflow tubing leading to a catheter snugly fitting the lower end of the common bile duct. The lower end of the bile duct was then perfused through this system with normal saline solution at constant pressure from a Marriott bottle. Tracings of pressures and flow were recorded on a Grass polygraph before and after complete division of the intestine at the pylorus.

RESULTS The relationship of flow through the sphincter of Oddi to gastric peristalsis was again clearly demonstrated (Fig. 1). After division of the bowel in the pyloric region and closing the duodenal stump with sutures, this characteristic pattern of flow through the sphincter could no longer be demonstrated, although gastric peristalsis was recorded as in the earlier part of the experiment. In addition some increase in resistance at the sphincter was apparent, as the perfusion pressure had to be raised from $10 \mathrm{~cm} \mathrm{H}_{2} \mathrm{O}$ to $20 \mathrm{~cm} \mathrm{H}_{2} \mathrm{O}$ to produce approximately the same flow rate.

\section{EXPERIMENT 2}

The pressure in the common bile ducts of conscious dogs was measured over long periods by adapting the method of Parry, Hallenbeck, and Grindlay (1955). With full aseptic precautions under anaesthesia, laparotomies were carried out in seven dogs. Polyethylene tubes (PE 100) were inserted into the common bile duct and brought out through the liver substance and then subcutaneously to the back of the neck. Two animals had a cholecystectomy

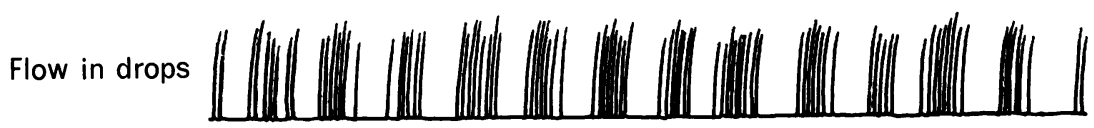

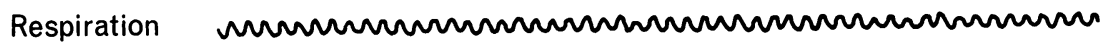

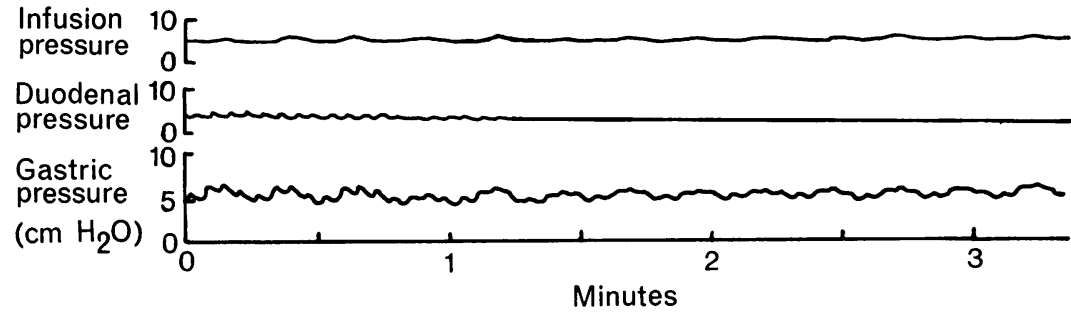

FIs. 1. Flow through the sphincter of Oddi and pressure changes in the gastric antrum. Each vertical line of the upper tracing indicates a drop falling through the drop chamber and a similar quantity of saline passing through the sphincter. Episodes of flow are related to gastric pressure changes but not to respiration or duodenal pressure changes. This flow pattern could not be demonstrated after gastroduodenal disconnexion. 
at the same time. The animals were allowed to recover from operation and trained to lie still on their abdomens for long periods. After at least a week prolonged pressure tracings were obtained by way of these tubes from the common bile ducts using a pressure transducer writing out on a Grass polygraph at the slowest paper speed. The dogs were starved for 24 to 48 hours before the observations commenced and fed during the period of observation which lasted from four to six hours. In two animals, after at least three satisfactory periods of recording, a Polya gastrectomy was done and after convalescence further tracings of bile duct pressure were obtained. In one cholecystectomized and one gastrectomized animal latex balloons were sutured to the lumen of the stomach and duodenum and pressures were recorded from similar long polyethylene tubes brought out of the viscus, across the abdominal cavity, and subcutaneously to protrude from the back of the neck. In two other animals a coeliac ganglionectomy was done after suitable tracings were obtained.

Altogether 43 four-to six-hour recordings were obtained from these seven animals. At least three tracings were available taken at intervals of two to three days before and after each manipulation.

RESUlTS Common bile duct pressures measured over long periods in this way in otherwise normal or cholecystectomized dogs have been described by previous authors (Menguy, Hallenbeck, Bollman, and Grindlay, 1958; Potter and Mann, 1926). The present observations confirmed their findings and will not be discussed in detail. The three types of waves seen by Potter and Mann were $(a)$ those associated with respiration (these can be seen superimposed on the larger wave form in Fig. 2); (b) a characteristic slow, steady rise in pressure over a number of minutes followed by a sharp fall and then a further steady rise; this may represent a long period during which the sphincter is closed followed by a short period of relaxation. In the present experiments the pressure was seen to rise between 3 and $13 \mathrm{~cm} \mathrm{H}_{2} \mathrm{O}$ over periods of seven to 40 minutes and then to fall quickly to the original level; (c) a wave having a frequency of five to $17 /$ minute. The faster frequencies were often seen and are similar in rate to the rhythmic contractions of the sphincter. The slower frequencies may have been confused with the waves now described.

It was found that in every intact animal, after taking food, but rarely otherwise, a very typical regular fluctuation of 2 to $5 \mathrm{~cm} \mathrm{H}_{2} \mathrm{O}$ was seen to occur quite regularly two to four times a minute (Fig. 2). This is at a rate typical of gastric peristaltic waves seen in previously reported acute experiments and this synchrony was confirmed in tracings from the intact animal with an intragastric balloon (Fig, 3).

No definite change in the pattern or mean pressure values was observed in any animal after destruction of the coeliac ganglion. After gastrectomy no change in mean pressure was detected. The two to four waves per minute were not seen on any occasion but otherwise the pressure patterns were similar to those seen in intact animals.

\section{DISCUSSION}

There is no doubt that a connexion between the
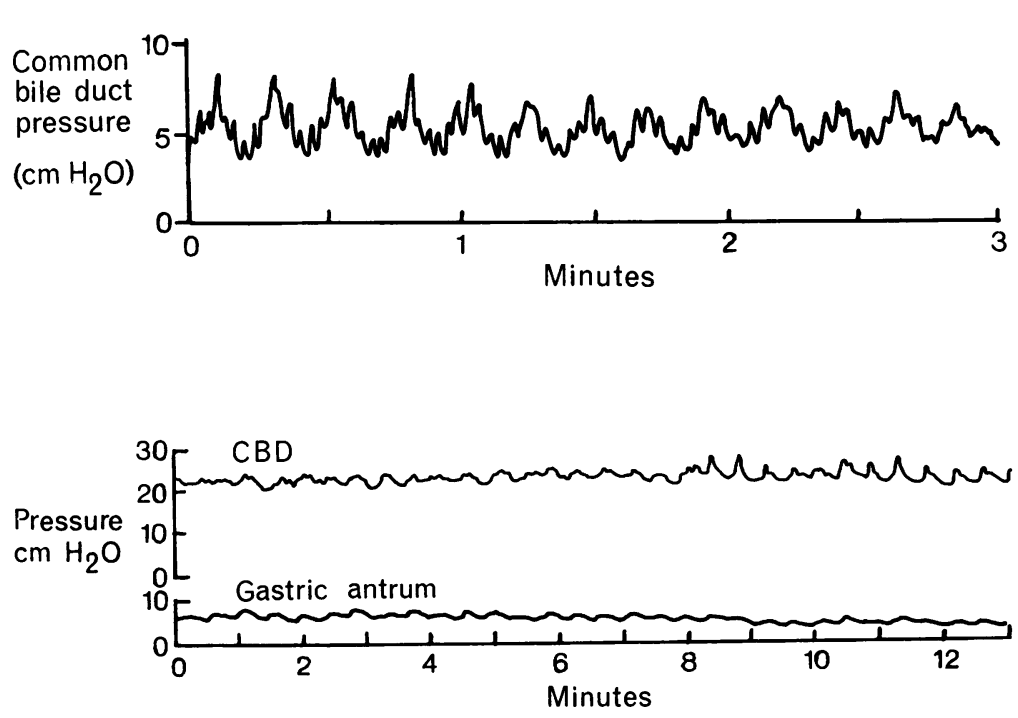

FIG. 2. Pressure changes in the common bile duct of an intact conscious dog after food. The main features are of a similar periodicity to those seen in the acute experiment illustrated in Fig. 1 and are therefore probably related to gastric peristalsis. This is an undamped trace and the smaller fluctuations are due to panting respirations. The regular major fluctuations could never be demonstrated after gastrectomy.

FIG. 3 Relationship of common bile duct pressure to antral pressure changes in a conscious dog after feeding. This is a damped and prolonged trace showing an obvious correlation of frequency of the two wave patterns. This relationship could not be demonstrated after Polya gastrectomy. (This animal had had a cholecystectomy which explains the relatively high biliary pressure.) 
resistance at Oddi's sphincter and gastric peristalsis exists, and, as these experiments have shown, that it is broken by gastroduodenal disconnexion. If, as has been suggested in the previous article, gastric peristalsis in the presence of food is associated with an inhibition of tone at the sphincter one might expect to find a degree of biliary hypertension after division of the alimentary tract at the pylorus. This was suggested in the first experiment reported but not confirmed by the long-term experiments. It seems certain that the waves seen at two to four per minute in bile duct pressure recordings in fed dogs were related to gastric peristalsis resulting in relaxation of the sphincter of Oddi, allowing bile to flow into the duodenum, especially so as these were not seen after a Polya type of gastrectomy.

The inability to observe biliary hypertension after gastrectomy in the long-term experiments may have been due to an adaptation of sphincteric action or to the presence of an intact gall bladder keeping the pressure low by its reservoir action. It is unfortunate in retrospect that the gall bladders were not removed from the gastrectomized animals.

These experiments confirm a relationship between gastric peristalsis and biliary dynamics and suggest that there may be a degree of functional obstruction at the sphincter of Oddi after gastrectomy. This may help to explain the increased incidence of biliary tract disease associated with the operation (Bockus, $1965)$ as due to induced biliary stasis.

It is possible that some of the symptoms of fullness, pain, and nausea experienced by patients after gastrectomy result from this mechanism. Conversion of a Polya gastrectomy to a Billroth I reconstruction often results in relief of symptoms of this sort (Tanner, 1966), and would be expected to restore the relationship between the two smooth muscle organs.

\section{SUMMARY}

Earlier work has indicated a relationship between the tone of the sphincter of Oddi and gastric peristalsis, especially if food is in the stomach. Evidence is now presented from both acute and long-term animal observations indicating that disconnecting the stomach from the duodenum, as occurs with a Polya type of gastrectomy, results in changes in the dynamics of bile flow into the duodenum.

I wish to thank Professor J. Englebert Dunphy for the opportunity and encouragement to carry out this study which was done in the Department of Surgery, University of California School of Medicine, San Francisco.

This work was supported by USPHS grant AM 09181 and was carried out while the author was in receipt of a Wellcome research travel grant.

\section{REFERENCES}

Bockus, H. L. (1965). Cholelithiasis. Ch. 113, Gastroenterology, Vol. 3, 2nd ed., p. 761. Saunders, Philadelphia and London.

Menguy, R. B., Hallenbeck, G. A., Bollman, J. L., and Grindlay, J. H. (1958). Intraductal pressures and sphincteric resistance in canine pancreatic and biliary ducts after various stimuli. Surg. Gynec. Obstet., 106, 306-320.

Parry, E. W., Hallenbeck, G. A., and Grindlay, J. H. (1955). Pressures in the pancreatic and common ducts: values during fasting, after various meals, and after sphincterotomy; an experimental study. Arch. Surg., 70, 757-765.

Potter, J. C., and Mann, F. C. (1926). Pressure changes in the biliary tract. Amer. J. med. Sci., 171, 202-217.

Tanner, N. C. (1966). Disabilities which may follow the peptic ulcer operation. Proc. roy. Soc. Med., 59, 362-368.

Wyatt, A. P. (1967). The relationship of the sphincter of Oddi to the stomach, duodenum and gall-bladder. J. Physiol. (Lond.), 193, 225-243. 